\title{
TIBF NEW EVIDENCE ON THE EFFECT OF CBOE OPTIONS LISTING ON THE VOLATILITY OF NEW YORK LISTED STOCKS
}

\author{
Khelifa Mazouz
}

Aston University, United Kingdom

\begin{abstract}
This paper re-examines the evidence on how the listing of options impacts on underlying stock's volatility by taking into consideration the possible presence of a learning effect, along with the impact of the very endogenous nature of the options listing decision itself. Our analyses are centred on both the portfolio approach as well as the individual stock approach applied on the sample of optioned stocks with a matched control sample. The results show that the individual stock approach yielded accurate results, as it is amenable to both the sign and the statistical significance test of variance change. However, unlike the individual stock approach, the more frequently applied portfolio approach relies more on the sign rather than the statistical significance. Based on these analyses, we found no evidence of the CBOE-option listing effect's presence on the volatility of the underlying stocks in the New York Stock Exchange.
\end{abstract}

JEL Classification: G14, G20 \& G21

Key words: Financial institutions, Cost efficiency, Profitability, Firm size.

\section{Introduction}

Whether listing options on an underlying stock stabilises or destabilises the underlying share's market price has been of interest to both regulatory bodies and market players since the initiation of the CBOE' ${ }^{1}$ first stock option contract. The topic is a continuous source of research focus for academic literature. Several theoretical models have been developed along with the adoption of different, empirical methodologies, investigating the implications of stock options trading regarding the pricing of the underlying shares.

${ }^{1}$ The first option contract was listed and traded on the Chicago Board Options Exchange (CBOE) on the $26^{\text {th }}$ of April 1973. At a much later date, options were also listed and traded within the New York Stock Exchange. 
Academic theory generally focuses on the concepts of market completeness and market efficiency to evaluate the effect derivative-securities trading has on the existing spot financial markets. The market can be made more complete if the newly introduced financial instruments accomplish certain objectives that could not have been accomplished by existing ones. Moreover, the market can become more efficient if the innovation can accomplish the same end as the existing products but at a lower transaction cost. In a complete market - with trivial transaction costs and trading constraints - stock options are regarded as redundant securities, as their payoffs can be synthesised by combining a position in the underlying stock with risk-free lending or borrowing. ${ }^{2}$

However, in the real world, where dynamic trading strategies replicating option payoffs are infinitely costly, introducing options can be useful in attaining efficiency in competitive equilibrium by expanding the set of contingencies covered by marketed assets (Ross, 1976). This gain in efficiency may still be jeopardised if the options listing makes the underlying stock more volatile (Chaudhury and Elfakhani, 1997). Theoretically, the impact of options listing on the underlying stock is controversial depending upon the assumptions that were initially made regarding the underlying market structure (Harris, 1989).

Options may convey a certain demand for securities and remove the uncertainty regarding the cost of obtaining an option-like payoff. The revealed information from the option trading by investors may affect the volatility of the underlying stocks (Grossman, 1988). Similarly, Dybvig and Ingersoll (1982) argued that the introduction of options would destroy traditional mean-variance pricing if market participants had a heterogeneous, utility function. The Dybvig and Ingersoll argument implied that the expected returns on both the options and their underlying stocks would differ from those predicted by CAPM. Cox (1976) claimed that in the context of futures trading, derivative securities attracted an additional group of traders that based their decisions on different information sets than those used by stock market participants before the introduction of derivatives. Thus, the stability or instability of the stock market after the options trading would depend on the accuracy of the new information. For example, a stabilising effect is produced if new information provided a better prediction of the spot price and vice versa. The volatility of the underlying stocks may also increase or decrease depending on whether options attract hedgers (Gorton and Pennacch, 1993) or speculators (Stein, 1987). Further, Ma and Rao (1988) contended that there existed a dual impact of options on the volatility of the underlying stocks. They argued that volatile stocks become more stable after listing because of the hedging behaviour of the uninformed traders. This resulted in stable stocks becoming more volatile after listing because of the speculative behaviour of the informed traders.

${ }^{2}$ The first option contract was listed and traded on the Chicago Board Options Exchange (CBOE) on the $26^{\text {th }}$ of April 1973. At a much later date, options were also listed and traded within the New York Stock Exchange. 
These theoretical positions show that the impact of options listing on the volatility of the underlying stocks is ambiguous. Because of this theoretical debate, the question about whether options trading stabilises or destabilises the market for the underlying assets is probably best addressed empirically. Using data from the U.S. market, the evidence from previous empirical studies is still mixed. A number of empirical studies - Trennepohl \& Dukes (1979), Whiteside et al. (1983), Klemkosky and Manes (1980), Bollen (1998), and Mayhew and Mihov (2000) showed that listing options had no significant effect on the volatility of the underlying stocks. However, Bansal et al. (1989), Conrad (1989), Skinner (1989) and Damodran and Lim (1991) provided empirical evidence that listing options reduced the volatility of the underlying stocks. There was at least one study (Rao and Ma, 1987) with evidence of a significant number of stocks experiencing higher volatility after options were traded.

This paper seeks to further examine the analysis of Ma and Rao's findings (1988). More volatile stocks become less volatile because of the hedging effect of the uninformed traders, where as less volatile stocks should become more volatile because of the speculative behaviour of the informed traders. Unlike other studies in literature, we will attempt to obtain what we deem to be the cleanest possible sample by removing suspected factors that may cause a bias in our results. We employ a control sample methodology to account for the possibility that any change in the volatility of the underlying stocks after the options introduction is due to market-wide conditions and/or to the endogenous nature of the decision to list options. The possible effect of options listing is examined using both a portfolio approach (which is widely used in literature) along with an individual stock approach (which we believe is more likely to provide accurate results). ${ }^{3}$

The rest of the paper is structured as follows. Section 2 will discuss our research motivation. Section 3 will introduce our sample selection criteria along with some detail of the empirical methodology applied. In Section 4, a description of the data set and presentation of the empirical results will be provided. Lastly, section 5 will offer our concluding remarks, specifically stating that the findings indicate no destabilisation of pricing as a result of the options listing effect.

\section{Literature on option listing effect}

\subsection{The non-uniform effect of options listing}

According to Ma and Rao (1988), listing stock options do not have a uniform impact on the volatility of the underlying security. They argued that options

\footnotetext{
${ }^{3}$ Bollen (1998) used the individual stock approach to examine the impact of options listing on the residual variance of the underlying stocks. He employed the approach with the assumptions of no beta changes and that the residuals are normally distributed. In this study, the individual stock approach is used to examine the impact of options listing on the total risk and on the residual risk, without the assumptions of Bollen.
} 
listing would increase the volatility of the stocks that were originally volatile, i.e., those stocks traded by uninformed traders. Listing would decrease the volatility of those stocks that were originally stable. The rationale behind their argument was that highly volatile stocks exhibited such volatility because of the large number of uninformed traders making transactions. The availability of the options market as an efficient hedging vehicle would attract the uninformed traders from the stock market to the options market. The ultimate result was a decrease in the volatility of the underlying stocks. Stable stocks are presumably less volatile because informed investors trade in them. High leverage and low transaction costs make options a useful vehicle for informed traders to capitalise on their information. Their speculative actions will in turn increase the noise in the stock market; thus, informed traders are more likely to trade in the options markets rather than the stock market. The resulting random noise in the options market is transmitted back to the stock market leading to an increase in the volatility of those stocks that were originally stable.

\subsection{Learning effect hypothesis}

Whiteside et al. (1983) and Detemple and Jorion (1990) argued that the options listing effect might not be stable over time. Market players might discover novel and diverse uses for listed options as time passes. This discovery might include new trading strategies involving options. It is also believed that the options listing effect might change over time following any future changes in regulations that govern the use of trading strategies involving options.

\subsection{The endogenous problem of options listing decision}

Another argument advanced by Skinner (1989) was that options listing was not a random event since options exchanges adopted stocks for listing that were rated highly on various attributes such as investors' interests, trading activity, and price volatility. Thus, if options exchanges deliberately listed options on stocks with mean-reversing volatility, one would expect the volatility to revert to its mean some time after the option initiation. Despite this attempt, Damodaran and Lim (1991) and Kamar et al. (1995) addressed the issue, claiming that the existing research had not adequately accounted for the endogeneity bias, which is the result of decisions made by the exchanges and regulators. They further argued that as long as the selection criteria changed with the changing market characteristics in the market, changes would coincide with option listings, even if the option listing itself had no impact on the underlying market.

Thus, if the proposition of Ma and Rao (1988) held, it could then be expected that the result of the impact of options listing on the underlying stock's volatility would depend on which type of stocks (stable or volatile) are dominant. If the sample chosen for a study included more volatile stocks than stable ones, the researcher would be more likely to conclude that options listing stabilises the 
market. The opposite conclusion would be made if the selected sample contained more stable stocks than volatile ones. However, if by chance a researcher selected a sample in which the decrease in volatility of volatile stocks is equal to the increase in volatility of the stable stocks, then a non-volatility effect of options listing would be the conclusion. Mayhew and Mihov (2000) provided evidence that this criterion was changing over time, meaning that exchanges were moving from listing more to less volatile stocks. This change in the listing requirements again implied that we would expect, on average, options listing to have a different impact on the volatility of the underlying stocks in different sub-periods during which the sample is selected.

Thus, we believe the answer as to whether options listing stabilises, destabilises, or has no impact on the volatility of the underlying stock lies in testing whether it is Ma and Rao's arguments and/or the endogenous nature of the options listing decisions that are responsible for the dependence of the empirical results on the sample type, sample size, and time horizon of the empirical study; or it is really that options listing is volatility-neutral with optioned stocks having similar behaviour to other stocks with similar characteristics.

\section{Sample selection criteria, data, and methodology}

\subsection{Sample selection criteria}

The aforementioned statements posed the idea that we were more interested in obtaining the cleanest possible sample rather than the biggest possible sample. Section 2 has shown why it might not be important to use the biggest possible sample in the study of options listing impact. This sub-section will further illustrate the way in which we construct what we determine to be the cleanest possible sample. In order to obtain the cleanest possible sample, we attempted to avoid any suspected problems that might cause bias in our results. We define at least four possible sources of bias that are linked to the sample selection. These might include market completeness, trading location, thin trading problems, and control sample.

The market completeness hypothesis can partly explain why including stocks with cross-listed options in the sample may cause a bias in the research results. The possible market completeness bias in the empirical literature on options listing was first recognised by Detemple and Jorion (1990). They examined the effect of option introductions on stock prices. They found significant increases in the total price for those options listed between 1973 and 1982 with no significant effect for the options introduced after 1982. The authors attributed the absence of the options effect after 1982 to the introduction of options on S and P 500 Futures on April 1982, which may have completed the market. This fact was ignored by a number of authors, including Ma and Rao (1986, 1988), Skinner (1989), Damodaran and Lim (1991), Fendenia and Grammatikos (1992), and Bollen (1998). 
Consider a given stock that has options listed on two different option exchanges. Including that stock in the sample on the basis of the latter option listing date might cause a problem, as the earlier listing for this stock in the market had probably been completed. Even if the stock was included on the basis of the earlier date, there is still a possibility of a sample selection bias if the cross-listing dates were close enough (i.e., the latter listing date is included in the analysis). The bias in this latter case might result from differences in the microstructure of the option exchanges.

Although there are no theoretical arguments why options should have a differential impact based on various trading locations, several authors (including Damodaran and Lim (1991) and Bollen (1998) suspected the trading location problem and they separated between stocks from different option exchanges and those from different stock exchanges. Being cautious, we accounted for the bias possibility that options have differential impacts in various trading locations, so we analysed only one stock market and one option market.

Similar to Chaudhury and Elfakhani (1997), precautions were also taken to avoid thin trading problems. The thin trading problem might be minimal in the earlier periods of options listing as exchanges listed mostly large and well-known stocks. However, the problem may be important in more recent periods being there is evidence of exchanges moving towards the listing of small and lowtrading volume stocks (Mayhew and Mihov, 2000). Thus, to avoid thin trading problems in our sample, we dropped all the events that do not have a complete data set. ${ }^{4}$ Because we attempted to compare the behaviour of the optioned stocks with those non-optioned stocks from the same industry, we also considered the availability of data for the best-matched pair of option and stock as another selection criterion. Market completeness, trading locations, and thin trading problems should also be taken into consideration when selecting control stocks from the same trading location as the optioned stocks.

\subsection{Testable hypotheses}

Driven by this aim, we test the following hypotheses:

H0: On average, there is no effect of options listing effect on the volatility of the underlying stocks.

The failure to reject $\boldsymbol{H} \boldsymbol{0}$ does not necessarily suggest that option listing is volatilityneutral. Some stocks may experience increases while others may experience decreases in the volatility; thus the overall effect may be neutral.

${ }^{4}$ Chaudhury and Elfakhani (1997) argued that to avoid the thin trading problem, those stocks with more than 20 missing, daily-price observations on either side of the options listing window should be excluded. 
Thus, we test the following hypothesis:

H1: Options listing have dual effect on the volatility of the underlying stocks. They increase the volatility of the relatively volatile stocks because of the hedging effect of uninformed traders, and they decrease the volatility of the relatively stable stocks because of the speculative effect of the informed traders (Ma \& Rao; 1988).

Again the failure to reject $\boldsymbol{H} \boldsymbol{1}$ does not necessarily suggest that the proposition of Ma \& Rao (1988) holds only for option listed stocks. In other words, the possibility that stable stocks in one period become more volatile in the next period while volatile stocks in one period become less volatile in the next period may be a market-wide phenomenon. This leads us to:

H2: Hypothesis H1 holds not only for optioned stocks, but also for nonoptioned stocks with similar characteristics.

If $\boldsymbol{H} \boldsymbol{2}$ is accepted, we can argue that options listing does not affect the volatility of the underlying stocks, otherwise a further investigation would be required.

\subsection{Returns and volatility measures}

Selecting the control sample and testing the above stated hypotheses require the calculation of daily returns. Following Hamill et al. (2002), we calculated the daily returns for stocks and the market index using the following formulas:

$$
\begin{aligned}
& y_{i, t}=\ln \left[\left(P_{i, t}+D_{i, t}\right) / P_{i, t-1}\right] \\
& x_{m, t}=\ln \left(P_{m, t}\right)-\ln \left(P_{m, t-t}\right)
\end{aligned}
$$

- $y_{i, t}$ and $x_{m, t}$ are the returns of stock $i$ and market index $m$ at time $t$;

- $P_{i, t}$ is the price of security $i$ on day $t$;

- $D_{i, t}$ is the total dividend paid during period $\mathrm{t}$;

- $P_{i, t}$ is the price of security $i$ at the end of the day $t-1$; and

- $P_{m, t}$ and $P_{m, t-1}$ are the prices of the market index on day $t$ and day $t-1$.

The, following Ma \& Rao (1988) and Kumar et al. (1995), we calculated the variance of returns for the underlying stocks, in both pre- and post-listing periods, by using the following variance formulas:

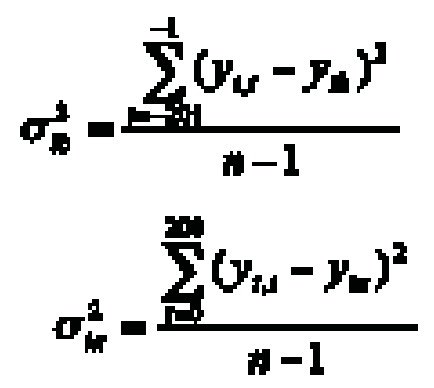



$-\boldsymbol{\sigma}$ is the variance of the return of stock $i$ before options listing;
$-\boldsymbol{\sigma}$ is the variance of return of stock $i$ after the option listing date; and
- yib and yia are the mean of returns in the pre-listing period and in the post-listing period for stock i, respectively.

Similar to others, we use the single index model to estimate the residual risk for each stock $i$. The single index model is:

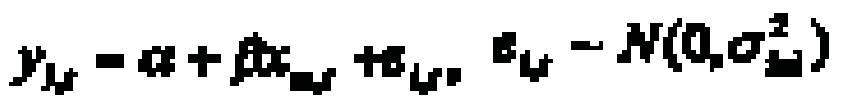

$-\sigma_{\boldsymbol{\omega}}$ is the residual risk for a given stock $i$; and

$\boldsymbol{\sigma}_{\boldsymbol{\omega}}$ takes two values depending on the estimation period of the single index model.

If the model is estimated in the pre-listing period (day -200 to day -1), then $\boldsymbol{\sigma}_{\boldsymbol{\sigma}}$ becomes $\boldsymbol{\sigma}_{\boldsymbol{a}}$. However, if the model is estimated in the post-listing period (day 0 to day +200$)$, then $\boldsymbol{\sigma}_{\boldsymbol{\omega}}$ becomes $\boldsymbol{\sigma}_{\mathbf{L}} \mathbf{s}$.

\subsection{Methodology}

Portfolio Approach: To begin the analysis of the effects of options listing on the underlying equity's volatility and for the empirical convenience, we constructed a portfolio of 234 observations. The portfolio contained 117 of the observations that represent the pre-listing return variance for each stock $i$ that is

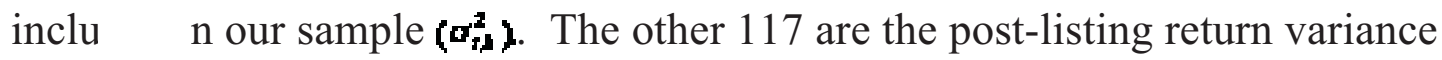
for the same stocks $\left(\boldsymbol{\sigma}_{\omega}^{\mathbf{2}}\right)$. We then created the following regression model: ${ }^{6}$

\section{$\sigma_{4}^{2}-a_{1}+a_{1} D U K_{r}+\eta \eta$}

- indicator $l$ takes two values: $b$ before option listing, and a after option listing;

- DUM $i$ is a dummy variable, taking a value of zero before options listing and unity after the options listing;

- $\eta$ is the random error term of equation (6);

- $\alpha_{0}$ is the average daily pre-listing variance; and

- $\left(\alpha_{0}+\alpha_{1}\right)$ is the average daily post-listing variance.

If options listing increases or decreases the return variance of the underlying stock, we would expect the coefficient on the dummy variable $\alpha_{1}$ to be positive (negative) and statistically significant. Otherwise, the equation would support hypothesis $H O$.

Then, we checked the results obtained from the equation (6) by applying the same equation to the portfolio of residual variances of the underlying stocks included in our sample as follows:

\footnotetext{
${ }^{5}$ As a reminder, the indicator ' $b$ ' means before listing and the indicator 'a' means afterlisting.

${ }^{6}$ The model construction principle is similar to Kumar et al. (1995) and Mendenhall \& Fehrs (1999).
} 


\section{$\sigma_{\omega}^{2}-\gamma_{0}+\gamma_{1} D U C_{1}+51$}

Again, it is the dummy variable's coefficient $\gamma_{1}$ that indicates the direction and the significance of the change in the volatility of the underlying stocks. If $\gamma_{1}$ is positive (negative) and statistically significant, equation (7) suggests that there is a significant increase (decrease) in the residual variance after options listing. Otherwise, hypothesis $\boldsymbol{H} \mathbf{0}$ is supported.

Following Skinner (1987) and Kumar et al. (1995), we checked the results using the Wilcoxon Signed Rank Test (WSRT). WSRT is used to test whether the return variance series (residual variance series) before options listing and the return variance series (residual variance series) after options listing are the same.

Accepting or rejecting hypothesis $\mathbf{H O}$ based only on the above tests does not actually end the task as it is argued that options listing may have a differential effect on different stocks (hypothesis $\boldsymbol{H 1}$ ). We use the same equation used by Ma and Rao (1988) to test hypothesis $\boldsymbol{H}$.

Ma \& Rao (1988) estimated the following equation:

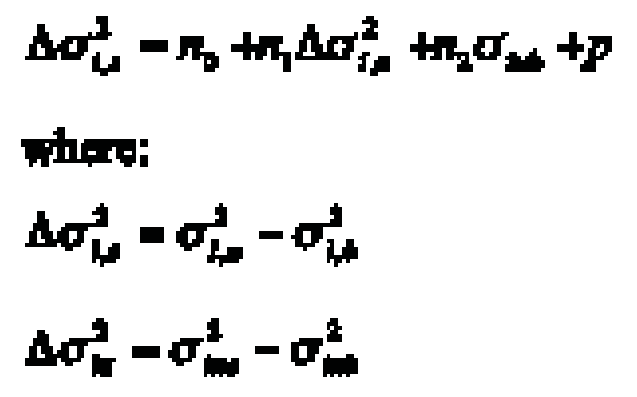

They argued that if options listing reduced the volatility of the more volatile stocks because of the hedging effect while increasing the volatility of stable stocks because of the speculation effect, we would then expect coefficient $n_{l}$ to be positive and coefficient $n_{2}$ to be negative. Thus, if the expectation of Ma \& Rao from equation (8) holds for our sample, then there is a possibility for the presence of a non-uniform effect of options listing. But, even if $\boldsymbol{H} \boldsymbol{1}$ was confirmed, there could be still the possibility that stocks with similar characteristics to our selected optioned stocks would show the same behaviour, and the entire story would then be explained by the market-wide conditions (hypothesis $\boldsymbol{H 2}$ ). Therefore, unless $\boldsymbol{H} \mathbf{2}$ is rejected, there is no options listing impact on the volatility of the underlying stocks. The best way to test $\boldsymbol{H} \boldsymbol{2}$ is to possibly use a control sample methodology.

Control Sample Methodology: Control sample methodology is not new in the area of options listing. Bollen (1998) used the control sample methodology to test whether the behaviour of the residual variance between the pre- and post-options listing periods was an industry-wide phenomenon. Mayhew and Mihov (2000) also used a similar approach to test whether the behaviour of the variance of return between preand post-options listing periods could be explained by the endogenous nature of the options listing decisions. However, control sample methodology has never been used to test whether the Ma and Rao (1988) proposition could be an industry-wide phenomenon. 
Before employing the control sample methodology to achieve the above stated purpose, it is first important to illustrate the way in which the control sample is constructed. When constructing the control sample, we undertook the following procedure:

First, we matched each optioned stock with a group of potential control stocks selected from the same industry sector which,

(i) had options listed neither before nor a year after the option listing date, and

(ii) had complete daily price data available. (These selection requirements were used to avoid the possible biases arising from different trading locations, market completeness, and thin trading.)

Then, the best control stock for each option-listed stock (among the group of potential control stocks) was selected by employing a ranking approach (see Mendenhall and Fehrs, 1999) on pre-listing average size, pre-listing return variance, and pre-listing residual variance. The ranking approach was used three times. The first time, we considered a given optioned stock's pre-listing average size as a benchmark. We then ranked the potential control stocks according to how close their pre-listing average size was to the benchmark. We associated number 1 to the potential control stock with the closest pre-listing average size to the benchmark, number 2 with the potential control sample with the second closest prelisting average size to the benchmark, and so on.

We applied the ranking approach for the second (and the third) time by using each optioned stock's pre-listing return variance (residual variance) as a return variance (residual variance) benchmark, ranking the potential control stocks according to how close their pre-listing return variance (pre-listing residual variance) was to the return variance (residual variance) benchmark.

We then associated the ranking with numbers starting from 1 for the potential control stock with the closest pre-listing return variance (residual variance) to the return variance (residual variance) benchmark, number 2 to the potential control stock with the second closest pre-listing return variance (residual variance) to the return variance (residual variance) benchmark, and so on. Employing the ranking approach three times would end up with each potential control stock, of a given optioned stock, having three numbers associated with it.

The first number indicated how close the potential stock's average pre-listing size was to the pre-listing size of the optioned stock. The second (third) number indicated how close the pre-listing return variance (residual variance) of the potential control stock was to that of the optioned stock. For each optioned stock, we selected the best control stock, from its potential control stocks, as being that stock with the smallest sum of ranks. The selected control sample is the used to test the hypothesis $\boldsymbol{H} \mathbf{2}$ in the following manner:

First, we employed the same portfolio approach on the control sample to test if the volatility behaviour between pre- and post-options listing of the sample of optioned stocks and that of non-optioned stocks was similar or different. We 
then checked the results using another alternative portfolio approach which combined the analysis of both the sample of optioned stocks and that of non-optioned stocks. This alternative portfolio approach used both a $t$-test and a Mann-Whitney test to check whether the change (if any) in the volatility of the optioned stocks was the same (or different) as the one (if any) of the control sample.

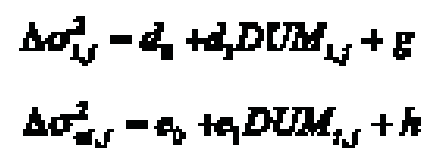

- $\boldsymbol{\Delta} \boldsymbol{\sigma}_{\mathbf{H}}^{\mathbf{A}}\left(\boldsymbol{\Delta} \boldsymbol{\sigma}_{\boldsymbol{\alpha}}^{\mathbf{2}}\right)$ represents the series of the change (post-listing minus prelisting) in the return variance (residual variance) of each optioned stock $\mathrm{i}$ and each control stock $\mathrm{j}$; and

- $D U M_{i, j}$ is a dummy variable, which takes a value of 1 if the stock is optioned and a value of 0 if the stock is not optioned. It is the sign of coefficient $\mathrm{d} 1$, e1 that indicates whether the change in the volatility of the optioned stock is higher or lower than the change in the volatility of the control sample.

A $t$-test was then used to check the significance of the difference. As a further check of the results, we performed a Mann-Whitney test to see whether the change in the volatility of options listing was significantly different from the change in the volatility of the control sample. We then checked the Ma andRao (1988) proposition that options listing had a dual effect dependent upon the volatility of the stocks when using equation (8). If all the above tests showed that there was a similar change (if any) between pre- and post-options listing periods in the volatility for both samples, and if Ma and Rao's equation (8) held in a similar way for both samples, then we could more confidently accept hypothesis $\mathrm{H} 2$. Accepting $\mathrm{H} 2$ means that options listing had no impact on the volatility of the underlying stocks.

Individual Stock Approach: Although the portfolio approach is widely used in the literature of options listing, we argue that the individual stock approach is more likely to provide accurate results. However, using only the sign of the change (or the ratio of the change) as an indicator for an increase or decrease in the volatility without checking the significance of the change may provide us with misleading results. Only a few authors have used the individual stock approach to test the possible volatility impact of options listing.

The individual stock approach is pursued using Merton's (1980) variance approximation. Merton (1980) argued that the $\operatorname{var}(\mathrm{Rt})$, where $\operatorname{var}(\mathrm{R} t)$ is the variance of the return $R$ at time t, approximately equals $E(R t 2)$, where $E(R t 2)$ is the expected value of the squared return at time $t$, for short measurement intervals. ${ }^{7}$ Using this argument, we examined the impact of options listing on the individual stocks' total risk and residual risk by applying the following equations to each stock: 


\section{$E\left(y_{i, j}^{2}\right)-\rho_{i p}+\rho_{i, p} D U M_{t}+\psi_{i}$ \\ $E\left(\varepsilon_{i, j}^{2}\right)-\pi_{i 4}+\pi_{i \alpha} D U M,+\xi i$}

- $\boldsymbol{E}\left(\mathbf{y}_{t, 1}^{\mathbf{2}}\right)$ is the squared daily returns of the stock i in the 400-day window,

- $\boldsymbol{B}\left(\boldsymbol{\sigma}_{i, 2}^{\mathbf{2}}\right)$ is squared daily residual, as estimated from the single index model, for each stock i; and

- The sign of the dummy coefficien $\boldsymbol{p}_{x \boldsymbol{\mu}}\left(\boldsymbol{s}_{\mu}\right)$ indicates the direction of the change in the return variance (residual variance) of the security $i$.

A t-test can then determine the significance of the change. We also used the Wilcoxon Signed Ranks Test to examine whether daily pre-listing variances and daily post-listing variances for each stock (both from the sample of optioned stocks and the control sample) belonged to the same distribution.

\section{Data and empirical results}

\subsection{Data}

The CBOE provided us with a list of option-listed stocks along with their corresponding dates of listing. The list involves all the stocks for which options have been introduced, within all the US options exchanges, from April 1973 to March 2001. In order to avoid the problem of trading location together with the issue of market completeness associated with the sample selection bias, we only included those New York Stock Exchange (NYSE) listed stocks with options contracts first introduced on the Chicago Board Option Exchange (CBOE) whose listing were found nowhere else within a year. There are 358 stocks that fulfil the above requirements.

Using the daily price data from Datastream for the Standard \& Poor's 500 index (S\&P500), which is used as a proxy for the market index, we examined only those stocks with options listing after January 1980 (298 stocks). To be selected, a stock must have complete, daily, dividend-adjusted price data for 400 observations around the options listing dates (200 observations before and 200 days after options listing). This restriction is necessary to avoid thin trading problems. Data are then available for 142 stocks. Our sample shrinks to 117 stocks when the control sample is being considered.

The potential control stocks were identified by the first four digits of their Compustat SIC code. The first four digits of the SIC code provided for each optioned stocks a list of matching stocks within the same trading location and industry sector as this particular optioned stock. In order to avoid the biases that might be linked to the control sample selection, the potential control stock was rejected if it had an option listed either earlier, or less than one year later, than the sample stock's option introduction. The control stock is also rejected if it does

\footnotetext{
${ }^{7}$ Skinner (1989) also used Merton's (1980) approximation to estimate the pre- and post-option listing
} 
not have a complete set of 400 daily price observations. Then, a ranking approach (described in Section 3.5.2) based on the pre-listing average size, pre-listing variance, and pre-listing residual variance was used to select the best control stock from the group of potential control stocks, for each option-listed stock. The mean and the median of pre-listing size return variance and residual variance for both the sample of optioned stocks and that of the control sample are reported in Table 1.

\subsection{Empirical results}

The Optioned Stocks Results: Panel A of Table 2 shows the summary statistic for the mean and median change along with the percentage (number) of stocks which show decreases and increases in the return variance and residual variance, between the pre- and post-options listing periods. The sample of optioned stocks shows a mean increase of $3.59 \%$ and a median increase of $3.23 \%$. Based on the Fisher Sign Test of the difference between the post- and pre-listing return volatilities, $51.28 \%$ (60 out of 117) of the stocks experienced an increase, while $48.71 \%$ (57 out 117) showed decreases in the return variance after options listing. Panel A also shows that the residual risk has decreased by an average of $2.85 \%$ and a median of $4.98 \%$. Again, based on the sign of the change in the residual risk, 53.8\% (63 in number) of the stocks showed a decrease and 46.2\% (54 in number) experienced an increase in the residual risk after the options listing.

To test the significance of the change in the return variance and its components, Panel B of Table 2 shows the Ordinary Least Square (OLS) estimation of equations (6) and (7) when they are applied to the sample of optioned stocks. Panel B shows that the dummy coefficient of equation (6) takes a positive value, suggesting a possible average increase in the volatility of the underlying stocks after the options listing. However, a t-statistic of only $0.314(p$-value $=0.754)$ associated with the coefficient $\alpha_{\imath}$ suggests that the increase is not significantly different from zero. The negative value associated with the dummy coefficient $\gamma_{1}$ of equation (7) implies a possible decrease in the average residual risk, but the t-

Table 1: The characteristics of the sample of the optioned stocks and the control sample which were selected using the ranking approach, in the pre-options listing periods.

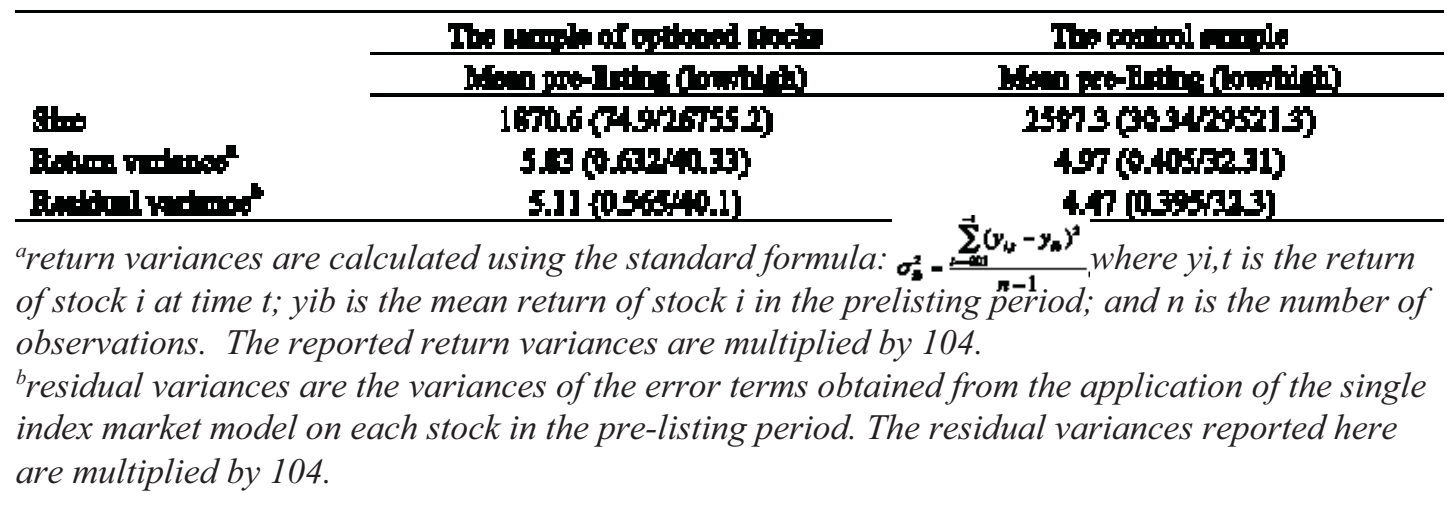


statistic of $0.042(\mathrm{p}$-value $=0.967)$ again indicates that this decrease is not statistically significant at the conventional confidence levels.

As a further check of the results, Panel C of Table 2 shows the result from the Wilcoxon Signed Ranks Test (WSRT). WSRT fails to reject the hypothesis that pre-listing return variances, residual variances, and betas are drawn from the same distribution as post-listing return variances, residual variances, and betas, respectively. The $z$-scores (and $p$-values) from the WSRT that is associated with testing the significance of the change in return and residual variances are, -0.271 (0.787) and $-0.366(0.715)$, respectively.

Thus, the results reported from the application of the portfolio approach seem to support the null hypothesis $\boldsymbol{H O}$. We argue that the results could be suspicious as our sample contains nearly an equal number of stocks that showed

Table 2: Summary statistic, regression technique, and non-parametric tests are used to test the possible impact of options listing on the volatility (variance of returns and residual variance) of options listing.

\begin{tabular}{|c|c|c|}
\hline \multicolumn{3}{|c|}{ 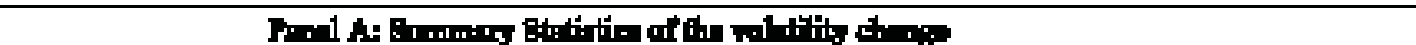 } \\
\hline & Voding of atiming & Retilini nilnow \\
\hline 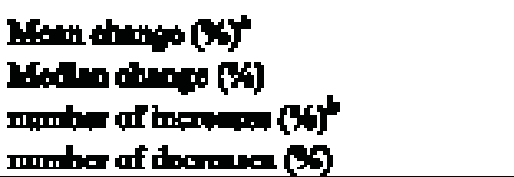 & 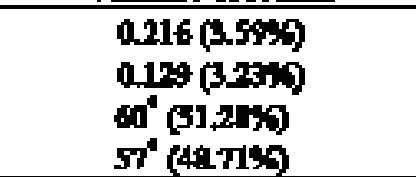 & 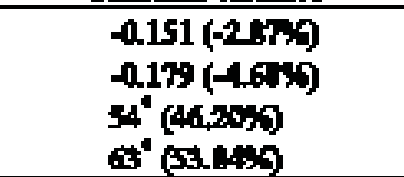 \\
\hline \multicolumn{3}{|c|}{ 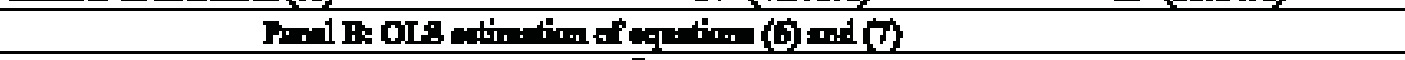 } \\
\hline & $\sigma_{i}^{2}-\alpha_{0}+\alpha_{1} D U M_{i}+m^{*}$ & $\sigma_{H}^{2}=\gamma_{0}+\gamma_{1} D U M_{f}+5^{d}$ \\
\hline 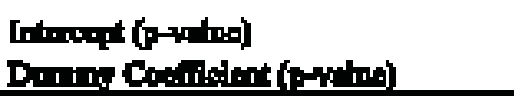 & $\begin{array}{l}0.000 \mathrm{~s} 1^{4}(0000) \\
2.160-05(0.754)\end{array}$ & 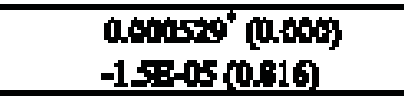 \\
\hline \multicolumn{3}{|c|}{ 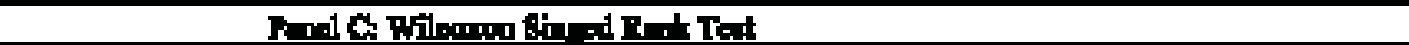 } \\
\hline & VAnos of nitur: & 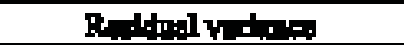 \\
\hline 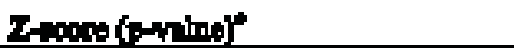 & $-0.27 L(0 . \pi n)$ & $0.36 .70 .715)$ \\
\hline \multicolumn{3}{|c|}{ 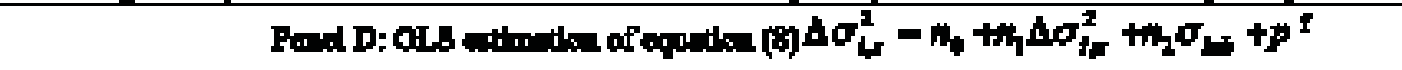 } \\
\hline 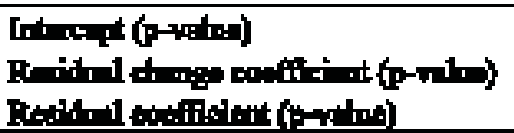 & $\begin{array}{l}3.906 \\
1.050 \\
-1.53\end{array}$ & $\begin{array}{l}\text { of (0.235) } \\
(0000) \\
5(0.049)\end{array}$ \\
\hline
\end{tabular}

* indicates the significance at the 1\% level

${ }^{a}$ the change is calculated as the difference between the post-and pre-listing variance; the reported mean and median change are multiplied by 104.

${ }^{b}$ the significance is based on Fisher Signed Test

${ }^{c} \boldsymbol{w}_{\mathbf{w}}^{\mathbf{2}}$ is the variance of return of each stock $i$, taking two values. One is the return variance before options listing, and the other is the return variance after the option listing. DUMi is a dummy variable that equals to zero in the pre-listing period and unity in the post-listing period.

${ }^{d} \boldsymbol{\sigma}_{\mathbf{w}}^{\mathbf{2}}$ is the residual variance of each stock $i$ and it takes two values from each stock. One is the prelisting residual variance and the other is post-listing residual variance.

e Wilcoxon Signed Rank test examined whether the pre-listing variances and the post-listing variances of the sample of optioned stocks belong to the same distribution.

${ }^{f} \mathbf{\Delta} \mathbf{w}$ denotes the difference between post-listing and pre-listing return variance of each stock $i$;

$\boldsymbol{\Lambda}_{i \mathbf{m}}^{2}$ is the change in the residual variance between post-and pre-listing periods for each stock $i$; and $\boldsymbol{\sigma}_{\boldsymbol{\omega}}^{\mathbf{z}}$ refers to the residual variance of stock $i$ in the pre-listing period. 
increase and those which experienced decrease in the return variance (and residual variance) after options listing. Thus, there is a possibility that options have a dual effect on the volatility of the underlying stocks. These effects are balanced out and averaged to zero.

Panel D of Table 2 shows the results of the OLS estimation of equation (8). The results from the OLS estimation of equation (8) are quite different from the expectations of Ma and Rao (1988). Similarly to Ma and Rao (1988), the coefficient $n_{1}$ from equation (8) is positive, equalling 1.050, and is statistically significant at the $1 \%$ level. This suggests a positive and statistically significant relationship between the change in the return variance and the change in the residual variance. However, in contrast to the findings of Ma and Rao (1988), we discovered no statistically significant relationships between the change in the volatility and the residual variance in the underlying stocks $\left(n_{2}=9.05 \mathrm{E}-05, \mathrm{p}-\right.$ value $=0.9489){ }^{8}$

We believe that these results are still logical for a number of possible reasons. First, Ma and Rao examined the early period (starting from 1973 and ending in 1984). We examined the more recent period (starting from 1980 until 2000). Because options listing is an endogenous decision and there is evidence that the listing criteria are changing over time (see Mayhew and Mihov, 2000), it is possible, therefore, that the characteristics of the stocks included in our sample differ from those included in Ma and Rao's (1988) sample. Also, the learning effect can play an important role here. Furthermore, it is possible that the market completeness hypothesis proposed by Detemple and Jorion (1988) holds. This means that the assumptions of Ma and Rao may hold in the earlier periods of options listing (1970s), but it does not hold for more recent periods (1980s and 1990s) as the market becomes complete.

Above all, there is also and possibility of further questioning the assumptions that were set forward by Ma and Rao (1988). Even following Ma and Rao's argument that options attract uninformed traders for hedging purposes and attract informed traders for speculation purposes, it is still possible that the residual error of any stock (stable or volatile) would either increase or decrease depending on how much stabilisation is gained from hedging and how much destabilisation is caused by speculation. Further, Ma and Rao's argument was based on the possible migration of informed and uninformed traders from the stock market to the options market. However, the availability of options as a hedging vehicle could actually attract more risk averse investors (those who believe that investing in stocks is too risky) to the stock market (Figlewski, 1986). This attraction, in turn, may further increase the volatility of those stocks that were originally more volatile (i.e., more uninformed traders making transactions). Also, following

${ }^{8}$ We also separately examined the period between 1980 and 1984 , and the results are still similar to those of the entire sample. However, we admit that the number of stocks included in this period is very small (6 companies). 
Cox's (1976) argument, options might attract a new group of traders to the options market and the volatility effect will depend on the accuracy of the information set of this new group of investors.

However, even after rejecting the hypothesis $\boldsymbol{H}$, which suggests a dual listing effect, we are still interested whether the ups and downs in the volatility of the stocks can be explained by the endogenous nature of the options listing decision and/or industry-wide conditions.

The Control Samples Results: Panel A of Table 3 shows the summary statistics of the average and median volatility change in the control sample after options listing. It also shows the percentage (a number) of the stocks that experienced increases and decreases in volatility based on the Fisher Sign Test. After options listing, the control sample shows that both the mean and the median

Table 3: Summary statistic, regression technique and non-parametric test to test the possible change in the volatility of the control stocks between pre- and post-options listing periods.

\begin{tabular}{|c|c|c|}
\hline \multicolumn{3}{|c|}{ 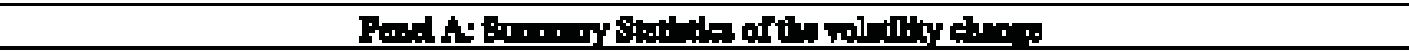 } \\
\hline & Voring of stom & 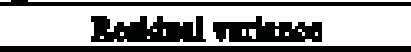 \\
\hline 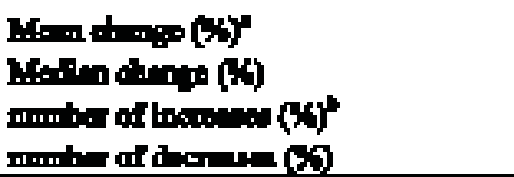 & $\begin{array}{l}0.467(9.415) \\
0.201(4.6256) \\
70^{\prime}(90.635) \\
47^{+}(40.17 \%)\end{array}$ & $\begin{array}{l}0.245(5.454) \\
0.100(9.30 \%) \\
60(51.75 \%) \\
54^{+}(45.15 \%)\end{array}$ \\
\hline \multicolumn{3}{|c|}{ 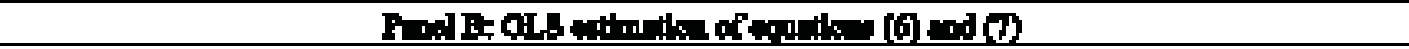 } \\
\hline & $\sigma_{i j}^{2}=a_{1}+x_{1} D U M_{i}$ & $\sigma_{H}^{2}=\gamma_{0}+\gamma_{1} D U M_{t}+5^{4}$ \\
\hline 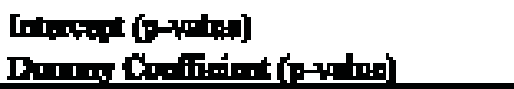 & 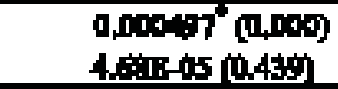 & $\begin{array}{l}0,000442^{4}(0,000) \\
2,45 \mathrm{E}-05(0,650)\end{array}$ \\
\hline \multicolumn{3}{|c|}{ 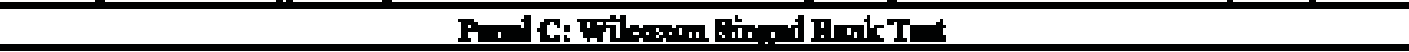 } \\
\hline & VAnsol of nimos: & 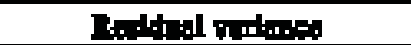 \\
\hline 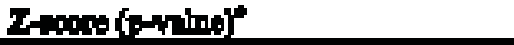 & $-1.0700 .06 \mathrm{D}$ & $-1.298(0.190)$ \\
\hline \multicolumn{3}{|c|}{ 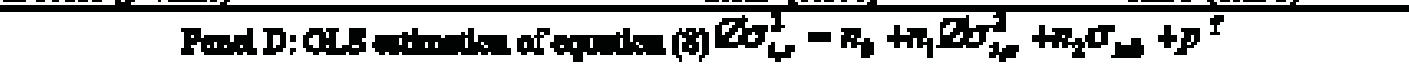 } \\
\hline 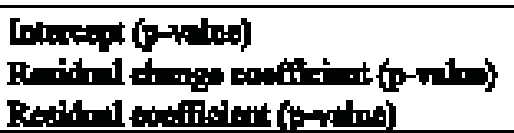 & & $(0.0433)$ \\
\hline
\end{tabular}

* and ** indicate significance at $1 \%$ and $5 \%$ levels, respectively.

${ }^{a}$ the change is calculated as the difference between post-and pre-listing variance. The reported mean and median changes are multiplied by 104.

${ }^{b}$ the significance is based on the Fisher Signed Test

${ }^{c} \boldsymbol{\sigma}_{\boldsymbol{z}}^{\mathbf{2}}$ is the variance of return of non-optioned stock $i$ and it takes two values. One is the return variance before options listing and the other is the return variance after the option listing. DUMi is a dummy variable which equals two zero in the pre-listing period and unity in the post-listing period.

${ }^{d} \boldsymbol{\sigma}_{\mathbf{G}}^{\mathbf{2}}$ is the residual variance of non-optioned stock $i$ and it takes two values from each stock. One is the pre-listing residual variance and the other is post-listing residual variance.

e Wilcoxon Signed Rank Test examined whether the pre-listing variances and the post-listing variances of the control sample belong to the same distribution.

${ }^{f} \boldsymbol{A \sigma}_{1}^{\mathbf{2}}$ denotes the difference between post-listing and pre-listing return variance of non-optioned stock $i$; $\boldsymbol{\Lambda}_{\boldsymbol{i}}^{2}$ is the change in the residual variance between post-and pre-listing periods for nonoptioned stock $i$; and $\mathbf{z}$ refers to the residual variance of non-optioned stock $i$ in the pre-listing period. 
of the return volatility increased by $9.41 \%$ and $4.62 \%$, respectively. Similarly, after options listing, the mean and the median of the residual risk increased by $5.54 \%$ and $3.30 \%$, respectively. $40.17 \%$ (47 in number) of the stocks' total volatility had decreased after options listing, with the remaining 59.83\% (70 in number) experiencing total volatility increases after options listing.

Similarly, the $46.15 \%$ (54 in number) of stocks experiencing a decrease in the residual volatility is a little lower than $53.75 \%$ (63 in number) of those with an increased residual volatility after the options listing.

Panel B of Table 3 shows the results of the OLS estimation of equations (6) and (7) applied to the control sample. The dummy coefficient $\alpha_{1}$ of equation (6) equals $4.68 \mathrm{E}-05$ with a $t$-statistic of $0.776(p$-value $=0.439)$. The dummy coefficient $\gamma_{1}$ from equation (7) is $2.45 \mathrm{E}-05$ with a t-statistic equalling 0.449 ( $p$ value of 0.654 ). Thus, the results from equations (6) and (7) suggest that there is no statistically significant change in the total and the residual volatility between the pre- and post-options listing periods.

Similar to the analysis applied on the sample of optioned stocks, the results of the possible change in the volatility of the control sample between the pre- and post-options listing periods are also checked using a Wilcoxon Signed Rank Test (WSRT). Panel B of Table 3 reports the results. WSRT supports the regression results of no volatility change suggested by equations (6) and (7). The Z-score from the WRST equals $-1.829(\mathrm{p}=0.067)$ for the total volatility change between the pre- and post-options listing period and is significant only at the $10 \%$ level.

Table 4: Comparison between the sample of the optioned stocks and the control sample in terms of the change in the return variances and residual variances between preand post-options listing periods.

\begin{tabular}{|c|c|c|}
\hline \multicolumn{3}{|c|}{ 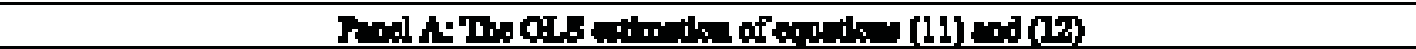 } \\
\hline & $\Delta \sigma_{h f}^{2}=d_{t}+d_{1} D U \Delta S_{d v}+g$ & $\Delta \sigma_{4}^{2}-\sigma_{4}+a_{1} D U A_{4}+h^{b}$ \\
\hline 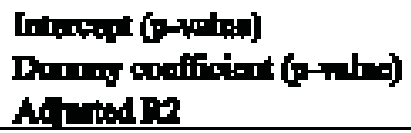 & 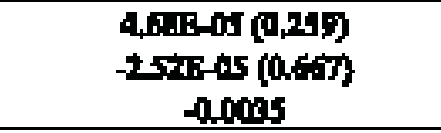 & $\begin{array}{l}-0,031(0,15) \\
0.431(0,319) \\
-1,40.05\end{array}$ \\
\hline \multicolumn{3}{|c|}{ 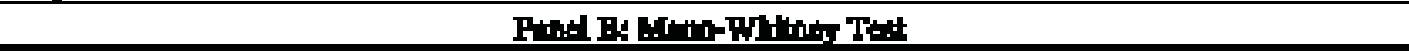 } \\
\hline & 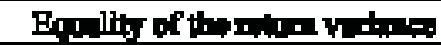 & 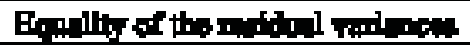 \\
\hline$z \operatorname{mon}\left(p-p_{i n}\right)^{4}$ & $-0.922[0.360)$ & $-1.432(0.152)$ \\
\hline
\end{tabular}

${ }^{a} \boldsymbol{\Lambda} \boldsymbol{\sigma}_{\mathbf{w}}^{\mathbf{2}}$ denotes the variance change between pre-and post-listing periods. $\mathbf{\Delta} \boldsymbol{\sigma}_{\mathbf{w}}^{\mathbf{v}}$ takes two values. One is $\mathbf{A v}_{\mathbf{j}}$, which is the variance change between pre-and post-listing for the optioned stock $i$. The other one is $\boldsymbol{\Delta \sigma}_{\mathbf{j}}^{\mathbf{2}}$, , which is the variance change between pre-and post-listing periods for the control stockj. DUMi,j is a dummy variable that takes the value of unity if the stock is optioned and value of zero if the stock is optioned.

${ }_{b} \mathbf{\Delta} \sigma_{\mathbf{u}}^{\mathbf{2}}$ refers to the change in the residual variance. $\mathbf{\Delta}_{\mathbf{u}}^{\mathbf{z}}$ also takes two values. One is the change in the residual variance of the optioned stock $i$, and the other is the change in the residual variance of the control stock $j$.

${ }^{c}$ Mann-Whitney test compares whether the series of the changes in the variance (and residual variance) of the sample of optioned stocks and the control stocks are the same. 
Also, the Z-score of -1.293 ( $p$-value $=0.196$ ) fails to reject the hypothesis that the residual variance series of the pre- and post-options listing periods belong to the same distribution.

The OLS estimation results of equation (8) on the control sample are reported in Panel $\mathrm{C}$ of Table 3. The application of equation (8) to the control sample provides us with similar results to the application of the same equation on the sample of optioned stocks. However, this time the coefficient of the $\mathrm{n} 2$ residual error is positive and statistically significant at the $1 \%$ level.

Results from the combined analysis: While the above section treated the sample of optioned stocks and the control sample separately, suggesting that the behaviour of both samples are very similar, this section combines the analysis of both samples, checking the results. Table 4, Panel A reports the results of the estimation of equations (11) and (12), which test whether the change in the sample of optioned stocks and that of the control sample are the same.

The dummy coefficient of equation (11), $d_{1}=-2.52 \mathrm{E}-05$, takes a negative value, suggesting that the increase in the total volatility of the sample of optioned stocks is less than that for the control sample. However, a $t$-statistic of $-0.431 \quad(p$-value $=$ 0.667 ) associated with $d_{1}$ suggests that the difference in the total volatility change between the two samples is not significantly different from zero. Similar results are also concluded when equation (12) is estimated. The negative sign of the dummy coefficient $e_{1}$ (equals $-3.96 \mathrm{E}-05$ ) suggests that the residual variance increases more for the control sample than for the sample of optioned stocks.

However, this difference is not statistically significant, as the $t$-statistic associated with the coefficient $e 1$ is only -0.757 ( $p$-value $=0.450)$. A Mann-Whitney Test also supports the above results. Panel $\mathrm{C}$ of Table 4 shows $Z$-scores and $p$-values resulted from Mann-Whitney Test comparison of the total variance change between the sample of optioned stocks and the control sample, which are -0.922 and 0.356 , respectively. Similarly, the Z-score and $p$-value resulting from the Mann-Whitney Test (which compares the samples residual variance change) are -1.432 and 0.152 , respectively.

To make the results of the above portfolio approach comparable to those of the individual stocks approach, as reported below, we repeat all the above reported tests using squared daily returns (squared daily residuals) as a proxy for the daily return (residual) variance. The results are very similar and are not reported in detail. We also applied the portfolio approach in two different sub-periods (the period of 1980s and that of 1990s), and the results in both sub-periods are still supportive to the null hypothesis of no volatility change are caused by options listing. 9

Results From the Individual Stock Approach: So far the results from the portfolio approach fails to reject the null hypothesis $\boldsymbol{H} \mathbf{0}$, which suggests that options

${ }^{9}$ The null hypothesis of no variance change is also supported when we divide our sample into a number of arbitrary chosen sub-samples. 
Table 5: The application of individual stock approach to test the significance of the change in the volatility of the underlying stocks between pre- and post-options listing periods.

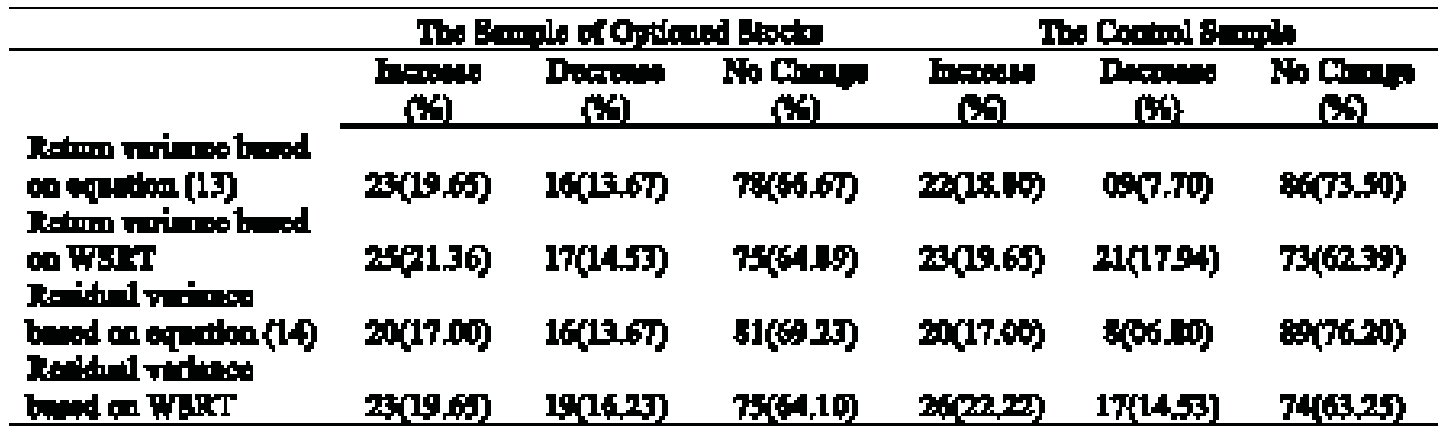

$a, b$ the number and percentage of the increases and decreases are significant at the $1 \%$ and $5 \%$ levels.

${ }^{c}$ equation (13) regresses the daily squared returns of each stock on the dummy variable, taking the value of zero before options listing and unity after options listing. The coefficient of the dummy variable measures the change in the variance between the two periods. If the coefficient is statistically significant, the change in the variance is also statistically significant.

${ }^{d}$ Wilcoxon Signed Ranks Test (WSRT) compares the change of the daily squared returns of each stock between the pre-and post-listing periods.

are volatility neutral. In the portfolio approach, the volatility effect of options listing depends on a large extent to the sign, rather the statistical significance of the change in the individual stocks' variances. Dependence only upon the sign can be a misleading approach as the volatility of any stock (or even the index) in the market will either show a positive or a negative relative change when estimated across two different periods (even with the same length). Thus, it is possible that the results reported above are simply a chance event. The options listing may actually cause a significant change in the volatility of the individual optioned stock, but the changes in the volatility of the individual non-optioned stock counter-part is not significant. It just so happens that the average and the median of the changes are the same. This possibility is examined by the application of the individual stock approach. The results are presented in Table 5 .

Before describing the results, it is important to pinpoint that the conventional $1 \%$ and $5 \%$ significance levels are used to indicate the statistical significance. Table 5 shows that, based on the statistical significance of dummy coefficient, of equation (13), $66.67 \%$ (78 in number) of the optioned stocks did not show a statistically significant change in the return variance after options listing; whereas only 23 $(19.65 \%)$ stocks showed a significant increase, with 16 (13.67\%) experiencing a significant decrease in the return variance after options listing. Similar results are also indicated by the WSRT, where $64.89 \%$ (75 in number) of the stocks showed no change, while $21.36 \%$ ( 25 in number) showed an increase with $14.53 \%$ (17 in number) experiencing decreases in the return variance after options listing.

Table 5 also shows the results of the application of the equation (14) and the WSRT on the residual variance of the individual optioned stocks included in our 
sample. The null hypothesis of no change in the residual variance of the individual optioned stocks is rejected $69.23 \%$ (81 out of 117 ) and $64.10 \%$ (75 in number) of the time based on the statistical significance of the dummy coefficient, , of equation (14) and the WSRT, respectively. Equation (14) indicated that 17\% (20 in number) of the stocks experienced a statistically significant increase while $13.67 \%$ of the stocks showed a significant decrease in the residual variance after the options introduction. Similarly, the WSRT indicates that $19.65 \%$ (23 in number) of the stocks showed a statistically significant increase while $16.23 \%$ (19 out 117) showed a statistically significant decrease in their residual variance after options listing.

The results of the individual stock approach on the control sample are broadly similar to those reported from the individual stock approach on the sample of optioned stocks. The results are also reported in Table 5. Based on the dummy coefficient, of the equation (13), 26\% (31 in number) of the control sample's stocks show statistically significant changes in the return variance after options listing. Among these stocks, $18.8 \%$ (22 in number) showed a significant increase with only $7.7 \%$ (9 in number) showing a significant decrease in the return variance after listing. However, the majority of the stocks in the control sample, 73.5\% (86 in number), experienced no statistically significant change in the variance after options listing.

The WSRT also fails to reject the null hypothesis that there is no change in the individual stock return variance, $62.39 \%$ (73/117) of the time. Based on the WRST, only $19.65 \%$ (23 of 117 ) of stocks showed a significant increase while $17.94 \%$ (21of 117) showed a significant decrease in the return variance after options listing. Similar results are indicated while equation (14) is applied on the test of the null hypothesis that there is no change in the residual variance of the individual stocks included in the control sample after options listing. Based on the statistical significance of the dummy coefficient, of equation (14), the null hypothesis of no change in the residual variance of the individual stocks after options listing is rejected $76.2 \%$ ( 89 out of 117 ) of the time. The remaining $23.8 \%$ (28 out 117 ) of cases are made up of $17 \%$ (20 of 117) of stocks with significant increases in the variance after options listing and $6.8 \%$ (8 out 117) with significant decreases in the residual variance after options listing. Again, the WSRT also supports the regression results, suggesting that $63.25 \%$ (74 out 117 ) of the stocks do not show a significant change in the residual variance after options listing. The WRST suggests that there are $22.22 \%$ (26 in number) of stocks that show a significant increase while $14.53 \%$ (17 in number) of stocks experience a significant decrease in the residual variance after options listing.

We also applied the individual stock approach on the sub-sample of the stocks that were listed in the 1980s and that of the stocks listed in the 1990s. The results also support the null hypothesis of no volatility change caused by options listing. ${ }^{10}$

\footnotetext{
${ }^{10}$ Similar results are also found when the individual stock approach is applied on a number of arbitrary.
} 
Therefore, the individual stock approach strongly supports the null hypothesis $\mathrm{H} 0$ that options listing is volatility-neutral. This is not only because the majority of optioned stocks in our sample did not show a statistically significant change in the return variance and the residual variance after the options introduction, but, also because the behaviour of the return variance (and the residual variance) between the pre- and the post-options listing periods is nearly the same for both the optioned stocks and their control stocks.

Being that we used the period starting from the 1980s on, the argument of market completeness can be used against our findings, i.e., the market became complete after 1970s, and therefore, did not show an impact thereafter. However, if the market has become complete and options listing do no longer have an effect on the underlying stocks' volatility, then the issue is no longer of concern to the regulatory agencies and market participants. Furthermore, there are still other studies, such as that by Bollen (1998) and Mayhew and Mihov (2000), which covered the entire period (starting from 1973 to 1996) of options listing and found no evidence of options listing on the volatility of the underlying stocks. Both Bollen and Mayhew dan Mihov also showed that the change of the volatility of the optioned stocks is very close to that of the control sample in different sub-periods.

\section{Summary and Conclusions}

For some time, the possible effect of options listing on the volatility of the underlying stocks has been of great concern by the popular press, regulatory bodies, traders, and academics. Because of the ambiguous nature of the theoretical debate in the area, the question whether options stabilise or destabilise the volatility of the underlying stocks is presumably best addressed empirically. The empirical evidence in the area is in itself mixed - some studies document increases, others decreases, and some argue for no change in the volatility of the underlying stocks after options listing. In 1988, Ma and Rao provided a theoretical model along with empirical results, which indicated the possibility that options listing may have a dual effect on the volatility of the underlying stocks, reducing the volatility of the more volatile stocks and increasing that of the stable stocks. Ma and Rao's argument, particularly when it is combined with the endogenous nature of the options listing decisions, suggests that the mean (and even the median) may not be a correct measure of the volatility change between the pre- and post-options listing periods.

This study had two purposes. The first was to provide new empirical evidence on the area of options listing. The second was to re-examine the argument proposed by Ma and Rao. In other words, our second purpose was to test whether Ma \& Rao's argument held only for the optioned stocks or whether it also applied to nonoptioned stocks with similar characteristics. Driven by these objectives, we undertook the following analysis. We first selected the cleanest possible sample of optioned stocks and matched it with another comparable sample of non-optioned 
stocks. Then, using a portfolio approach that involved both regression techniques and non-parametric tests to examine the significance of the change in the volatility (both total and residual) of the underlying stocks. The portfolio approach was applied on both samples, separately and as a combination. We then estimated the Ma \& Rao model on both samples. Finally, we employed the individual stock approach, which we believed to be more accurate on all the stocks in both the sample of optioned stocks and the control sample.

The results of the application of the portfolio approach suggest that there is no statistically significant change in the volatility of the underlying stocks, either the optioned and non-optioned ones, after options listing. We also find no evidence of the dual effect of options listing in that the results of the application of the Ma and Rao model, where our samples differ from Ma and Rao's expectation. We do not interpret our results as spurious for at least two reasons. First, we believe that the assumptions made by $\mathrm{Ma}$ and Rao are questionable. Second, market completeness could be a reason for the difference between our results and those of Ma and Rao. As a further check for the possibility of a chance event, which may be resulted from the application of the portfolio approach, we examined the statistical significance of the change in the volatility of each of the underlying stocks in both samples. The individual stock approach supports the null hypothesis that options listing are volatility-neutral. This is not only because the majority of the optioned stocks show no significant volatility change after options listing, but also because the behaviour of the volatility of individual stocks in both sample is very similar.

Acknowledgement: The author acknowledges the useful comments of an anonymous reviewer and editorial help of the editorial desk on the submitted version of the paper. The author's e-mail contact is k.mazouz@aston.ac.uk; Phone: (4412) 12043041.

Author statement: Khelifa Mazouz is an academic staff at the Aston Business School, Birmingham, United Kingdom. The anonymous reviewers' comments are acknowledged with thanks. The editors of the Journal made significant changes to the original version of the paper, for which we record our sincere appreciation. The errors are the responsibility of the author.

\section{References}

Bansal, V., Pruitt, S., and Wei, K., 1989. An empirical reexamination of the impact of CBOE option initiation on the volatility and trading volume of the underlying equities: 1973- 1986. Financial Review 24, 19-29. 
Black, F., and Scholes, M., 1973. The pricing of options and corporate liabilities. Journal of Political Economy, 637-654.

Bollen, N., 1998. A note on the impact of options on stock return volatility. Journal of Banking \& Finance 22, 1181-1191.

Chaudhury, M. and Elfakhani, S., 1997. Listing of put options: is there any volatility effect?. Review of Financial Economics 6, 57-75.

Conrad, J., 1989. The price effect of options introduction. Journal of Finance 44, 487-498.

Cox, C., 1976. Futures trading and market information. Journal of Political Economy 48, 1215-1237.

Damodran, A., and Lim, J., 1991. The effects of options listing on the underlying stocks' return processes. Journal of Banking and Finance 15, 647-664.

Detemple, J., and Jorion, P., 1988. Options listing and stock returns. Fist Boston working paper no. 89-13, Columbia University.

Detemple, J., and Jorion, P., 1990. Option listing and stock return: an empirical analysis. Journal of Banking \& Finance 14, 781-801.

Dybvig, P., and Ingersoll, J., 1982. Mean-variance theory in complete markets. Journal of Business 55, 233-251.

Fendenia, M., and Grammatikos, T., 1992. Options trading and the bid-ask spread of the underlying stocks. Journal of Business 65, 335-351.

Figlewski, S., 1986. The interaction between derivative securities on financial instruments and the underlying cash markets: an overview. Journal of Accounting, Auditing \& Finance, 299-318.

Gorton, G., and Pennacchi, G., 1993. Security baskets and index-linked securities. Journal of Business 66, 1-28.

Grossman, S., 1988. An analysis of the implication for stock and return price discovery in stock, futures, and options markets. Journal of Futures Markets 16, 353-387.

Hamill, P., Opong, K., and McGregor, P., 2002. Equity option in the UK: a comparison of market-based research methodologies. Journal of Empirical Finance 9, 91-108.

Harris, L., 1989. S\&P500 cash stock price volatilities. Journal of Finance 44, $1155-1175$.

Kemkosky, C., and Manes, S., 1980. The impact of options on the underlying securities. Journal of portfolio Management (Winter), 12-18.

Kumar, R., Sarin, A., and Shastri, K., 1995. The impact of index options on the underlying stocks: the evidence from the listing of Nikkei Stock Average options. Pacific-Basin Finance Journal 3, 303-317.

Ma, C., and Rao, R., 1988. Information asymmetry and option trading. The Financial Review 23, 39-51.

Mayhew, S., and Mihov, V., 2000. Another look at option listing effects. Working Paper, http://www.ssrn.com 
Mendenhall, R., and Fehrs, D., 1999. Option listing and stock price response to earnings announcements. Journal of Accounting \& Economics 27, 57-87.

Merton, R., 1980. On estimating the expected return on the market: An explanatory investigation. Journal of Financial Economics 8, 323-361.

Ross, S., 1976. Options and efficiency. Quarterly Journal of Economics 90, 7589.

Skinner, D., 1989. Options markets and stock return volatility. Journal of Financial economics 23, 61-78.

Stein, J., 1987. Information externalities and welfare reducing speculation. Journal of Political Economy 95, 1123-1145.

Tennepohl, G., and Dukes, W., 1979. CBOE options and stock volatility. Review of Business and Economic Research 14, 49-60.

Whiteside, M., Marry, M., Dukes, P., and Dunne, P., 1983. Short term impact of option trading on underlying securities. Journal of Financial Research 6 , 313-321. 37 